\title{
O impacto na qualidade de vida dos pacientes geriátricos com vertigem no contexto da APS: uma revisão bibliográfica
}

\author{
The impact on the quality of life of geriatric patients with vertigo in the context of PHC: a \\ bibliographic review
}

El impacto en la calidad de vida de los pacientes geriátricos con vértigo en el contexto de la APS: una revisión bibliográfica

Silvio Matheus Azevedo Rocha1*, Ana Beatriz de Starling Hudson Soares², Ana Beatriz Molina Ramos $^{3}$, Debbie Priscila Weber ${ }^{4}$, Diovana Amazonas Danuta Andrade da Silva ${ }^{5}$, Fhilipe Hollanda Cavalcanti Soares ${ }^{4}$, Giovana Cordeiro de Moraes $^{6}$, Ingrid Albuquerque Araujo Gomes Self ${ }^{7}$, Sabrina Jéssica Pedrosa Ribeiro ${ }^{8}$, Gabriela Gonçalves Campos ${ }^{9}$.

\section{RESUMO}

Objetivo: O presente artigo faz uma análise do impacto na funcionalidade e o grau de incapacidade que as doenças vertiginosas provocam nos idosos e como isso influencia na autonomia e na qualidade de vida destes indivíduos, no contexto da Atenção Primária da Saúde. Revisão bibliográfica: O estudo esclarece que a tontura é uma das doenças mais recorrentes na clínica médica e que a probabilidade de ser acometido por esse sintoma aumenta devido à senilidade, visto que sua prevalência é maior que $50 \%$ nos idosos acima de 85 anos. Outra configuração pertinente é que a tontura se expressa de diversas formas, sendo a vertigem a mais frequente. Todavia, avalia-se a dificuldade de diagnóstico das doenças que causam a vertigem, pois os pacientes não conseguem expressar o que estão sentindo, fazendo com que cerca de $40 \%$ destes pacientes não sejam diagnosticados corretamente. Considerações finais: Dessa maneira, o correto manejo desse quadro se faz necessário para que a qualidade de vida dos idosos seja mantida. A literatura ainda é insuficiente sendo fundamental uma maior atenção da comunidade científica.

Palavras-chave: Idosos, Vertigem, Atenção primária à saúde.

\begin{abstract}
Objective: This article analyzes the impact on functionality and the degree of disability that vertigo causes in the elderly and how it influences the autonomy and quality of life of these individuals, in the context of Primary Health Care. Literature review: The study clarifies that dizziness is one of the most recurrent diseases in the medical clinic and the probability of being affected by this symptom increases due to senility, since its prevalence is greater than $50 \%$ in the elderly over 85 years. Another relevant configuration is that dizziness is expressed in different ways, with vertigo being the most frequent. However, the difficulty in diagnosing the diseases that cause vertigo is assessed, as patients are unable to express what they are feeling, causing about $40 \%$ of these patients not to be diagnosed correctly. Final considerations: Thus, the correct management of this condition is necessary for the quality of life of the elderly to be maintained. The literature is still insufficient, requiring greater attention from the scientific community.
\end{abstract}

Key words: Elderly, Vertigo, Primary health care.

\footnotetext{
1Universidade Tiradentes (UNIT), Aracaju - SE. *E-mail: silviomatheus@icloud.com 2Pontifícia Universidade Católica de Minas Gerais (PUC-MG), Betim - MG.

${ }^{3}$ Universidade Federal do Mato Grosso do Sul (UFMS), Três Lagoas - MS.

${ }^{4}$ Faculdade de Medicina do Vale do Aço (UNIVAÇO), Ipatinga - MG.

5Universidade Nilton Lins, Manaus - AM.

${ }^{6}$ Universidade Federal de Ouro Preto (UFOP), Ouro Preto - MG.

7 Universidade Ceuma, São Luís - MA.

${ }^{8}$ Faculdade de Minas (FAMINAS), Belo Horizonte - MG.

${ }^{9}$ Centro Universitário de Caratinga (UNEC), Caratinga - MG.
} 


\section{RESUMEN}

Objetivo: En este artículo se analiza el impacto en la funcionalidad y el grado de discapacidad que provoca el vértigo en las personas mayores y cómo influye en la autonomía y calidad de vida de estos individuos, en el contexto de la Atención Primaria de Salud. Revisión bibliográfica: El estudio aclara que el mareo es uno de los la mayoría de las enfermedades recurrentes en la clínica médica y la probabilidad de ser afectado por este síntoma aumenta debido a la senilidad, ya que su prevalencia es superior al $50 \%$ en los ancianos mayores de 85 años. Otra configuración relevante es que el mareo se expresa de diferentes formas, siendo el vértigo el más frecuente. Sin embargo, se valora la dificultad de diagnosticar las enfermedades que provocan vértigo, ya que los pacientes son incapaces de expresar lo que sienten, provocando que alrededor del $40 \%$ de estos pacientes no sean diagnosticados correctamente. Consideraciones finales: Por tanto, el correcto manejo de esta patología es necesario para mantener la calidad de vida del anciano. La literatura es aún insuficiente, lo que requiere una mayor atención por parte de la comunidad científica.

Palabras clave: Anciano, Vértigo, Atención primaria de salud.

\section{INTRODUÇÃO}

O idoso pode apresentar limitações que ocasionam declínios nos âmbitos biológico, psicossocial, socioeconômico, e cultural. Essas alterações impactam as relações interpessoais, a rotina, a independência e o próprio papel social dos idosos. Diante disso, a forma com que as pessoas de idade mais avançada lidam com as funções cotidianas também passa por adaptações, que podem ser agravadas por fisiopatologias, uma vez que as limitações do paciente senil os levam à fragilidade (SCARMAGNAN GS, et al., 2021; AGUIAR VFF, et al., 2019).

Dentre os distúrbios que acometem os idosos, a tontura é o sintoma mais habitual, sendo um motivo comum de consultas na atenção primária. Segundo Menant JC, et al. (2017), 10\% a 30\% dos idosos residentes na comunidade relatam sintomas atuais ou crônicos de tontura. Esse sintoma clínico que pode se tornar um evento incapacitante, pode culminar na diminuição do convívio social, interferir na qualidade de vida dos idosos, e desestruturar sua dinâmica familiar, levando ao crescimento do número de senis que necessitam de cuidados a longo prazo, podendo inclusive culminar na institucionalização dos mesmos. (PHILLIPS A et al., 2018; FERREIRA CJS, et al.,2021).

A manifestação da tontura pode ser agrupada em categorias, sendo elas: vertigem, pré- síncope/síncope, desequilíbrio e tontura inespecífica. Segundo Dommaraju S e Perera E (2016), a vertigem corresponde a mais de $50 \%$ das manifestações sendo descrita pelos pacientes como uma ilusão de auto movimento ou como se o ambiente estivesse se movendo, sendo essa sensação de movimento não real comumente chamada de sensação de giro. Grande parte desses quadros têm origem no sistema vestibular, sendo a Vertigem posicional paroxística benigna (VPPB) uma das mais comuns. Os demais casos têm relações com o sistema extravestibular (como alterações visuais, neuronais ou psíquicas), variações cardiovasculares, distúrbios metabólicos ou modificações no sistema musculoesquelético (RAMÍREZ SA, et al., 2018; RIBEIRO KF, et al., 2018; GUTIÉRREZ V, et al., 2020).

Embora existam causas vestibulares e não vestibulares de vertigem que possuem tratamento específico, grande parte dos sintomas vertiginosos são subdiagnosticados ou diagnosticados de forma incorreta, sendo desafiador a recomendação de terapia para tais sintomas na atenção primária, visto que é necessário a realização de um diagnóstico preciso para estabelecer tal terapia. (PHILLIPS A, et al., 2018).

O grau de incapacitação e de restrição de funcionalidade gerado pelas vestibulopatias podem impactar o estado psicológico do idoso, levando a quadros depressivos, de ansiedade e afetando a autoestima, dessa forma é imprescindível realizar um tratamento multidisciplinar que permita a manutenção da qualidade de vida do paciente, uma vez que é perceptível que a Vertigem impacta nos aspectos pessoais, sociais, mentais e funcionais desses indivíduos (GERAGBTY AW, et al., 2017; SILVA K, et al., 2020).

Devido à relevância desse tema para a população idosa, este estudo tem como objetivo fazer uma análise do impacto na funcionalidade e o grau de incapacitação que as doenças vertiginosas provocam nessa população e como isso influencia na autonomia e na qualidade de vida dessas pessoas. 


\section{REVISÃO BIBLIOGRÁFICA}

A Organização Mundial de Saúde (OMS) estimou que até meados de 2050 a quantidade de idosos se elevará exponencialmente. Dessa forma, é fundamental elaborar um sistema que esteja mais preparado para receber esse público que apresenta alterações no corpo e que não estão necessariamente relacionadas com doenças, mas sim que ocorrem devido à redução da vitalidade e a consequente fragilidade. Dentro disso, avaliações multidimensionais no local considerado porta de entrada do usuário, atenção primária à saúde são fundamentais, principalmente, no que tange aos casos de vertigem (MAIA LC, et al., 2020).

Ao envelhecer, o sistema nervoso central tem a sua capacidade em processar os sinais vestibulares, visuais e proprioceptivos prejudicada. As estruturas responsáveis pelas funções vestibulares costumam degenerar com a idade. Isso acontece porque as células ciliadas diminuem em cerca de 6\% por década. Ressalta-se que as células ciliadas no canal semicircular degeneram antes e em maior extensão em comparação às células ciliadas nas máculas dos otólitos (SCHERER S, et al.,2012; JAHN K, 2019).

Além da perda celular, foram demonstradas mudanças morfológicas e funcionais das células remanescentes. Estudos post-mortem mostraram que o declínio de células no gânglio vestibular (também conhecido por gânglio de Scarpa) começa a partir da terceira década, com acentuação a partir da sexta década em diante. Do ponto de vista funcional, os déficits relacionados à idade acontecem com mais frequência nos canais semicirculares, seguido pela função sacular, enquanto o utrículo permanece menos afetado (JAHN K, 2019; FERNÁNDEZ L et al., 2015).

Dessa maneira, as células ciliadas começam a degenerar precocemente e em uma constante ao longo da vida, em comparação ao nervo vestibular e aos neurônios, que iniciam o processo de degeneração a partir da quinta década de vida, aumentando com a idade. A perda das células ciliadas periféricas é compensada pelo aumento na sensibilidade das estruturas centrais (JAHN K, 2019).

A vertigem é provocada por disfunção no aparelho vestibular e apresenta-se com uma ideia distorcida do movimento, sendo comum ser associada ao tipo rotatório e a alguns outros sintomas, como náuseas, vômitos, zumbidos e hipoacusia. Deve-se diferenciá-la dos demais tipos de tontura (síncope, desequilíbrio e sensações cefálicas variadas) para isso investiga-se com perguntas ao paciente sobre se há sensação de que os objetos ou o ambiente estão girando ao seu redor, se a resposta for sim é vertigem objetiva, caso contrário, se tem a sensação de a cabeça estar girando: vertigem subjetiva (VINCENT AVV, et al., 2020).

Assim, ao identificar a queixa de tontura como vertigem, o passo seguinte é realizar o diagnóstico mais aprofundado para diferenciar se é vertigem periférica ou central. A vertigem periférica é mais frequente em idosos, a lesão é no labirinto e/ou no nervo vestibular, considerado o oitavo par craniano sendo responsável por funções sensitivas. Na vertigem central, o dano encontra-se no núcleo vestibular, cerebelo ou tronco cerebral, sendo considerada mais grave (GRILL E, et al., 2014).

Ao identificar clinicamente a diferença entre os tipos de vertigem, pode-se estabelecer diagnóstico e prognóstico da doença. Por ser a forma topográfica mais diagnosticada, a vertigem periférica pode estar acompanhada de prostração, sudorese, palidez e perda breve da consciência, além da associação de zumbidos e hipoacusia. O indivíduo normalmente inclina-se para o lado da lesão vestibular durante a queda apresenta instabilidade, mas que não chega a comprometer a marcha. Na vertigem central, não há presença de perda da capacidade auditiva, no entanto pode estar associada a outros sintomas neurológicos, como falta de coordenação dos movimentos, dificuldade de articulação da fala, visão dupla, perda de consciência, déficits focais sensitivos ou motores ou dos nervos cranianos, entre outros. Outra intercorrência envolve a marcha, em que é comum ocorrer retropulsão, mesmo com a vertigem sendo mais leve (RIBEIRO K, 2017).

A VPPB é uma das afecções causadoras de vertigem mais comuns. Geralmente ocorrem quando o paciente assume certas posições críticas da cabeça, principalmente quando vai se deitar ou levantar. Quando o paciente é examinado, não se tem evidências de alterações vestibulares e não se notam anormalidades auditivas. O diagnóstico é feito através da manobra de Dix-Hallpike. Essa manobra é realizada de modo a mover o paciente da posição sentada para a deitada com a cabeça colocada a $30^{\circ}$ abaixo do plano horizontal e voltada $45^{\circ}$ para um dos lados. Após alguns segundos, ao realizar a manobra, o indivíduo refere vertigens 
e observa-se o nistagmo, caracterizado como movimentos involuntários dos olhos que ocorrem de forma repetitiva e não são controlados, podendo resultar em uma visão reduzida do indivíduo. Esses movimentos podem ser mais ou menos bruscos e assumir várias direções. O mais comum é o nistagmo horizontal, que bate em direção ao pavilhão auricular e dura menos de 15 segundos. É causada pela liberação de material otoconial que migra para os canais semicirculares e os estimula erroneamente, sendo esta a causa mais frequente de vertigem (SOCIEDADE BRASILEIRA DE MEDICINA DE FAMÍLIA E COMUNIDADE, 2012).

No estudo de coorte feito por Vincent AVV, et al. (2020) com 417 idosos na atenção primária à saúde realizado durante 10 anos relacionou os tipos de tontura (pré-síncope, vertigem, desequilíbrio e outras formas) bem como suas causas, sendo mais vistas as doenças cardiovasculares e as vestibulopatias, em relação às quais possuíam o maior índice de mortalidade. Ocorreram 169 mortes no intervalo dado e a menor taxa de óbito foi em relação a forma de tontura tipo vertigem e a causa foi a vestibulopatia. Ainda no estudo de Vincent AVV, et al. (2020), a atenção primária é de extrema importância no tratamento de tontura, uma vez que cerca de $80 \%$ de indivíduos com tal patologia em países como Holanda, Reino Unido e Estados Unidos realizam a intervenção médica com profissionais desse nível da saúde e não com um otorrinolaringologista ou otoneurologista, o que evidencia a eficácia das consultas no nível primário sendo que por muitas vezes não é necessário um encaminhamento a um especialista.

Em um estudo transversal foi evidenciado que pacientes com vertigem geram, economicamente, a necessidade de maiores recursos financeiros tanto na atenção primária quanto na secundária. Um paciente com tal enfermidade gasta em média 818 euros a mais em relação à saúde por um ano se comparado a um indivíduo sem vertigem. Além disso, no que tange a licença médica, $57 \%$ dos pacientes com vertigem, durante um ano, obtiveram-na por cerca de 7 dias (WANG X, et al., 2019).

Dessa forma, um ponto que se faz necessário avaliar é sobre a dificuldade de diagnóstico das doenças que causam a vertigem, pois os pacientes não sabem relatar o que estão sentindo de modo exato, o que pode gerar muitas variáveis. Por conseguinte, como pelo menos $20 \%$ dos idosos apresentam certo espectro de fragilidade é fundamental o papel da APS nessa primeira linha de identificação dos distúrbios oto-neurológicos para evitar que o paciente frequente várias especialidades médicas, para que haja antecipação de possíveis agravos e redução de impacto na qualidade de vida. (MAIA LC, et al., 2020).

De acordo com Hamilton C e Christmas C (2020), menos de 50\% dos pacientes recorrem a outros níveis de atenção em caso de sentir tontura, além de que o diagnóstico pode ser alcançado com uma boa anamnese e exercício físico revelando que não é viável recorrer aos segundo e terceiro nível do Sistema Único de Saúde (SUS). Basicamente, um olhar mais atento às queixas do paciente permite que se alcance a motivação de procurar os cuidados da Atenção Básica, não sendo necessários exames complementares como os laboratoriais e de radiografia, que se mostram por vezes prejudiciais.

A não identificação da patologia e a perda gradual do potencial auditivo causa desconforto nos idosos, pois eles ficam sem entender o que está sendo conversado e por vezes são excluídos do diálogo. Tal situação afeta substancialmente o cotidiano dessa faixa etária causando prejuízos na qualidade de vida. Pontua-se também que o conceito de bem-estar refere-se a forma de se posicionar no contexto em que vive, a partir do viés social, mental e físico. Avalia-se a preocupação e perda de confiança, já que ocorre um maior comprometimento do cotidiano, com aumento do medo de perder o equilíbrio e sofrer com quedas. São fatores que levam a um maior isolamento por medo de andar pela rua (PETRI M, et al., 2017; MARTINS E SILVA DC, et al., 2016).

Segundo Geragbty AW, et al. (2017), idosos com mais de 70 anos possuem maior probabilidade de cair dentro de casa, enquanto os com menos têm chance de quedas fora do ambiente domiciliar. Tal situação torna os mais velhos vulneráveis, visto que aumenta a chance de fraturas e com isso a dependência de outras pessoas. Isso é visto, grande parte das vezes, como perda de independência e pode ocasionar sintomas depressivos envolvendo sentimentos negativos, dificuldade de concentração e baixa autoestima na população idosa.

Logo, com o envelhecimento da população e a presença de doenças nessa faixa etária surge a necessidade de desenvolver tratamentos adequados. Como a tontura e suas subcategorias compreendem 
um grupo de distúrbios mais prevalente entre a população com mais de 60 anos é importante descrever as intervenções terapêuticas que amenizam os sintomas relacionados a esses quadros, sobretudo no contexto da Atenção Primária à Saúde. Com isso, evidenciam-se ações que abranjam tanto o idoso fragilizado quanto os familiares para que haja inserção na Política Nacional Humaniza SUS e o cuidado seja seguido de modo mais eficiente. Ocorre então o vínculo paciente, família e equipe de saúde, tendo coordenação e integralidade do cuidado. (SOUSA FTL, et al., 2019).

A terapêutica para vertigem em idosos pode ser realizada de diferentes formas. Entre elas, destaca-se a fisioterapia, que inclui a execução de manobras e exercícios, além da medicação (RIBEIRO K, 2017). Como afirma Regauer V, et al (2020), o tratamento para as patologias associadas ao desequilíbrio devem fazer a utilização de exercícios como meio principal.

No contexto do tratamento por meio da fisioterapia, a Vertigem Posicional Paroxística Benigna, um dos principais tipos de vertigem, pode ser tratada, por exemplo, por Manobras de Reposicionamento de Otólitos (MRO). Os otólitos são estruturas presentes dentro de câmaras no aparelho vestibular, cujo deslocamento pode acarretar sintomas associados à vertigem. Desse modo, entende-se que o reposicionamento dessas estruturas ajuda a amenizar as manifestações clínicas da tontura. A Manobra de Epley é frequentemente a mais recomendada para pacientes diagnosticados com VPPB e deve ser executada por meio de comandos de movimento (RIBEIRO K, 2017).

Outra manobra útil em caso de vertigem periférica é a de Semont, em que o paciente na maca primeiro tem a cabeça rotacionada em direção ao ouvido não afetado e em seguida o corpo é abaixado em direção a maca, tal ação busca que o indivíduo fique deitado sobre o lado afetado, com o nariz para cima. Depois de três minutos, se movimenta para ficar do lado oposto, com o nariz para baixo, por três minutos. Após o tempo cronometrado, a pessoa volta a posição vertical e a cabeça volta para o normal (GEBHART I, 2021).

Além disso, existem outras intervenções não farmacológicas que auxiliam no tratamento da vertigem, como exercícios de Reabilitação Vestibular (RV). Um dos exemplos envolve situações simples como olhar para cima e baixo, de um lado para outro, concentrar em um dos dedos da mão com o braço esticado, além de flexão e extensão da cabeça. Na execução dessa série de exercícios, que consiste em atividades voltadas para a orientação do paciente por meio do movimento corporal, evidencia-se melhora na adaptação vestibular, de forma que a RV promove cura completa em aproximadamente $1 / 3$ dos pacientes (LOPES AL, et al., 2018)..

Em relação ao tratamento medicamentoso, as drogas mais utilizadas são antieméticos, anti-histamínicos e anti-inflamatórios. O uso desses remédios, embora relacionado ao controle dos sintomas de vertigem, por vezes, pode atrasar a cura da doença por não tratar seu cerne, mas sim apenas seus sintomas. Dessa forma, o diagnóstico baseado na sintomatologia pode ser atrasado, assim como a cura da vertigem (BALATSOURAS DG, et al., 2018).

Segundo Petri M, et al (2017), a neurite vestibular, doença de Ménière e VPPB (vertigem posicional paroxística benigna) apresentam maior probabilidade de melhora e assim manutenção de bem-estar para 0 paciente geriátrico, ao contrário das síndromes vestíbulo-cocleares. Corrobora-se também para o tratamento em tempo hábil do VPPB, enquanto a tontura crônica causa maior degradação ao cotidiano do indivíduo.

Por fim, cabe salientar que na APS, a realização das manobras supracitadas e a educação para que os pacientes geriátricos as realizem também em ambiente domiciliar são de extrema importância para o manejo da vertigem. A necessidade do tratamento farmacológico deve ser avaliada pelo médico responsável, de acordo com o quadro clínico de cada paciente (BALATSOURAS DG, et al., 2018).

\section{CONSIDERAÇÕES FINAIS}

O processo fisiológico do envelhecimento traz consigo mudanças que podem prejudicar a capacidade funcional dos idosos, sendo a tontura uma das principais queixas na Atenção Primária à Saúde. O diagnóstico precoce e a correta conduta terapêutica seja com o uso de manobras de correção ou seja com o uso de fármacos são extremamente necessários para preservar a qualidade de vida dos idosos. Diante deste quadro, 
fica evidenciada a necessidade de criação de protocolos que busquem padronizar métodos de prevenção, diagnóstico e tratamento da tontura no contexto da atenção básica.

\section{REFERÊNCIAS}

1. AGUIAR VFF, et al. Ação educativa com profissionais de saúde na identificação do idoso vulnerável: relato de experiência. Revista eletrônica Acervo Saúde, 2019; 28:e932.

2. BALATSOURAS DG, et al. Benign paroxysmal positional vertigo in the elderly: current insights. Clin Interv Aging. 2018; 13:2251-2266

3. DOMMARAJU S, PERERA E. An approach to vertigo in general practice. Aust Fam Physician, 2016;45:190-194.

4. FERREIRA CJS, et al. O cuidado ao idoso institucionalizado: perspectivas dos cuidadores e da equipe de enfermagem. Revista eletrônica Acervo Saúde, 2021;13(5): e7230.

5. FERNÁNDEZ L, et al. Vertigo and dizziness in the elderly. Frontiers in Neurology, 2015;6:1-6.

6. GRILL E, et al. Health services utilization of patients with vertigo in primary care: a retrospective cohort study. Journal of Neurology, 2014; 261: 1492 - 1498.

7. GUTIÉRREZ V, et al. Rehabilitación vestibular em personas mayores com disfunción vestibular. Rev. ORL, 2020; 11(1): 67-78.

8. GERAGBTY AW, et al. Internet-Based Vestibular Rehabilitation for Older Adults With Chronic Dizziness: A Randomized Controlled Trial in Primary Care. Annals of family medicine, 2017; 15(3).

9. GEBHART I, et al. Sémont Maneuver for Benign Paroxysmal Positional Vertigo Treatment: Moving in the Correct Plane Matters. Otology e neurotology, 2021; 42 (3): 341 - 347.

10. HAMILTON C, CHRISTMAS C. Evaluation and management of difficult symptoms in older adults in primary care. Medical clinics of North America, 2020; 104(5): 885-894.

11. JAHN K. The aging vestibular system: dizziness and imbalance in the elderly. Advances in Otorhinolaryngology, 2019;82: 143-149.

12. LOPES AL, et al. Evidências científicas da reabilitação vestibular na atenção primária à saúde: uma revisão sistemática. Audiol Commun Res. 2018;23:e2032.

13. MAIA LC, et al. Fragilidade em idosos assistidos por equipes da atenção primária. Ciência e saúde coletiva, $2020 ; 25$ (12): $5041-5050$.

14. MARTINS E SILVA DC, et al. Effects of vestibular rehabilitation in the elderly: a systematic review. Aging clinical and experimental research, 2016; 28 (4): 599-606.

15. MENANT JC, et al. Tailored multifactorial intervention to improve dizziness symptoms and quality of life, balance and gait in dizziness sufferers aged over 50 years: protocol for a randomised controlled trial. BMC Geriatrics, 2017;17:56.

16. PHILLIPS A, et al. Anticholinergic and sedative medications and the risk of vertigo or dizziness in the German primary care setting-A matched case-control study from the CONTENT registry. Pharmacoepidemiol Drug Saf, 2018;1-9.

17. PETRI M, et al. Health-related quality of life and disability in patients with acute unilateral peripheral vestibular disorders. Brazilian Journal of Otorhinolaryngology, 2017; 83 (6): 1-11

18. RAMÍREZ SA, et al. Diagnóstico de vértigo periférico para el médico de atención primaria. Revista Med, 2018;26(1).

19. REGAUER V, et al. Physical therapy interventions for older people with vertigo, dizziness and balance disorders addressing mobility and participation: a systematic review. BMC geriatrics, 2020; 20:494

20. RICCI NA, et al. Revisão sistemática sobre os efeitos da reabilitação vestibular em adultos de meia-idade e idosos. Brazilian Journal of Physical Therapy, 2010; 14: 1-9.

21. RIBEIRO KF, et al. Effectiveness of Otolith Repositioning Maneuvers and Vestibular Rehabilitation exercises in elderly people with Benign Paroxysmal Positional Vertigo: a systematic review Brazilian Journal of otorhinolaryngology, 2018; 84(1):109-118.

22. SOCIEDADE BRASILEIRA DE MEDICINA DE FAMÍLIA E COMUNIDADE. Tratado de Medicina de Família e Comunidade: Princípios, Formação, Prática. 1 ed. Brasil: Artmed, 2012; 1794-1796.

23. SOUZA FTL, et al. Projeto terapêutico singular: uma ferramenta de promoção da saúde do idoso. Revista eletrônica Acervo Saúde, 2019; (24), e659.

24. SCARMAGNAN GS, et al. A complexidade da tarefa afeta negativamente o equilíbrio e a mobilidade de idosos saudáveis. Revista brasileira de geriatria e gerontologia, 2021;24(1):e 200120.

25. SCHERER S, et al. Tontura em idosos: diagnóstico otoneurológico e interferência na qualidade de vida. Revista da Sociedade Brasileira de Fonoaudiologia, 2012;17(2).

26. SILVA K, et al. Avaliação quantitativa e qualitativa do equilíbrio corporal em idosas ativas e sua relação com a saúde no geral. CoDAS, 2020;32(6):e20180246.

27. TAKANO NA, et al. Qualidade de vida de idosos com tontura. Brazilian Journal of Otorhinolaryngology, 2010;76(6).

28. VINCENT AVV, et al. Prognosis and survival of older patients with dizziness in primary care: a 10-year prospective cohort study. Annals of family medicine, 2020; 18:100-109.

29. WANG, X, et al. Vertigo and dizziness cause considerable more health care resource use and costs: results from the KORA FF4 study. Journal of neurology, 2019; 266: 2120-2128. 